\title{
The motion simulation of three-DOF parallel manipulator based on VBAI and MATLAB
}

\author{
Zhuo Zhen, Chaoying Liu* and Xueling Song \\ Institute of electrical engineering, Hebei University of Science and Technology, Shijiazhuang, Hebei, \\ 050000, China \\ liucy@hebust.edu.cn
}

Keywords: Machine vision, the three-DOF parallel manipulator, VBAI, MATLAB.

Abstract. In order to truly reflect the object situation of catch and place of the three-DOF parallel manipulator based on machine vision, and on the basis of the modeling of the trajectory and mechanical structure, this paper mainly focuses on the analysis on the manipulator system by using the co-simulation approach based on the MATLAB and VBAI software, with modeling and simulation of target acquisition, mechanical structure, and trajectory planning for manipulator system built. In addition to this, the application of machine vision technique has been proposed to sample and grasp the image of an object. The simulation results show that the manipulator system can meet to the requirements of the design accuracy and rapidity, which proves the effectiveness of the co-simulation method.

\section{Introduction}

Parallel manipulator has the advantages of high rigidity, high flexibility, strong carrying capacity, especially to achieve translational parallel mechanism has attracted intense attention ${ }^{[1-2]}$. The parallel manipulator based on Delta ${ }^{[3]}$ mechanism is the most representative. The IRB 340 Flex ${ }^{[4-5]}$ Picker is a kind of visual manipulators based on Detla parallel mechanism,w hich is developed by ABB company by Adpet technology . The robot can crawl disorderly placed objects. The robot can make use of image processing techniques for automatic sorting and crawl. Based on visual analysis of the robot has become a hot research. At present, domestic and foreign about parallel manipulator research mainly focuses on the mechanism design theory, kinematics, dynamics modeling and analysis, control strategy and so on. This paper presents a simulation method of motion of three degree of freedom parallel manipulator based on machine vision; the whole working process of parallel manipulator based on machine vision was complete simulation.

\section{Target acquisition and target positioning}

The robot use Vision Builder AI software for target acquisition and positioning. Vision Builder AI NI Introduces referred VBAI is a software product for visual inspection. This tool is an ideal tool to lab for a quick visual effect validation, and production line to achieve simple test ideal test platform. Target object image can be imported to the VBAI program by means of the camera. Taking mechanical parts as experimental object, we will import the image into the VBAI program, then the following treatment:

1) Gradation processing

First of all to gray-scale image processing, gray processing is to accelerate thereaction speed of software. Because we only need to get the object contour information, so does the need for color image. After gray image after processing as shown in Fig. 1;

2) Calibrate Image

Because of the basis of mechanical hand grasping objects is the actual distance, so we have to be the pixel value into the actual distance value. The actual distance of the system unit is $\mathrm{mm}$. Select two points on the image, and then enter the actual distance can be in software, distance calibration interface as shown in Fig. 2; 
3) Match Pattern

The role of MMM is to establish a template to capture objects, and then can be identified from the object test bench. Template matching the interface as shown in Fig. 3;

4) Set Coordinate System Setup

Locate the template matching the geometry center as the center coordinates for the target position, processing interface as shown in Fig. 4.

5) Coordinate values of output

Manipulator control system is set up coordinate system based on camera at the upper left of the area of origin. If you select the robot mechanical origin position for the upper left corner of the imaging area, it can be directly output target coordinates to the robot's motion controller. The coordinate output interface as shown in Fig. 5. 16.457 is the X-axis coordinate and Y-axis coordinate is 11.2952. $\mathrm{Z}$-axis coordinate is determined based on the shape of the target object and preset trajectory and can be set directly on the mechanical hand grasp program.

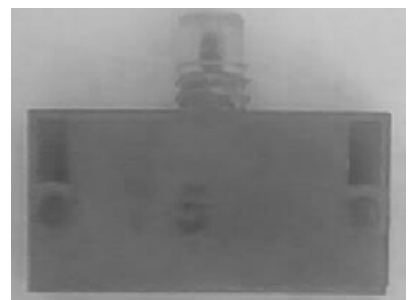

Fig. 1 Gradation processing

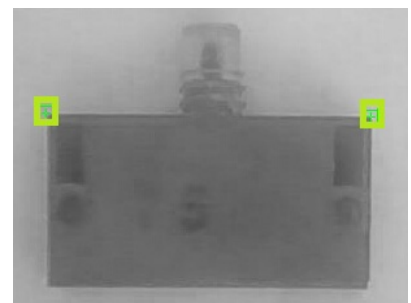

Fig. 2 Calibrate Image

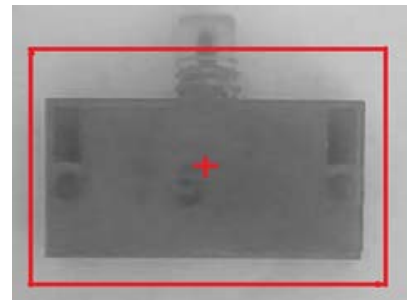

Fig. 3 Match Pattern

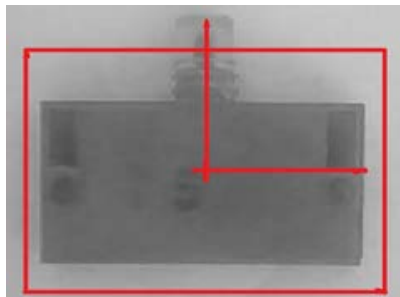

Fig. 4 Set Coordinate System Setup

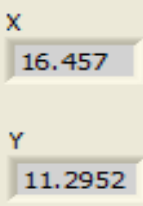

Fig. 5 Coordinate values of output

\section{The position inverse solution of three degree of freedom parallel manipulator}

The moving platform is regarded as a point $O^{\prime}$. Reference coordinate system $O$-XYZ based on the static platform Center $O^{\prime}$. The position vector of Moving platform reference point $O^{\prime}$ can be expressed as: $r=[\mathrm{x}, \mathrm{y}, \mathrm{z}]$. Eq. 1 indicates the position vector of point $O^{\prime}$.

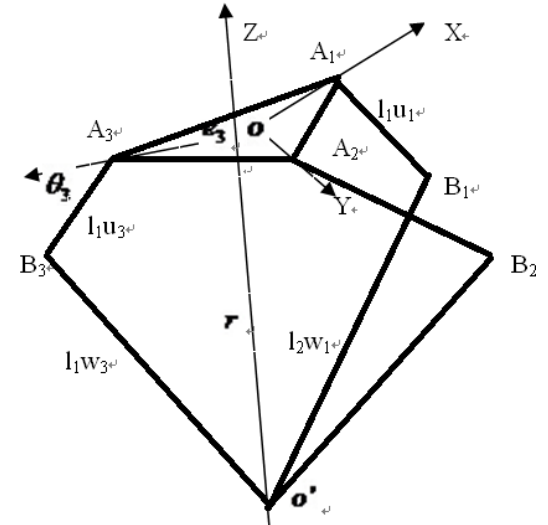

Fig.6The simplified geometry structure diagram

$r=e_{i}+l_{1} u_{i}+l_{2} w_{i}, i=1,2,3$

$e_{i}=e\left(\begin{array}{lll}\cos \beta_{i} & \sin \beta_{i} & 0\end{array}\right)^{T}$

$\beta_{i}=(i-1) \frac{2 \pi}{3}-\frac{\pi}{6}$

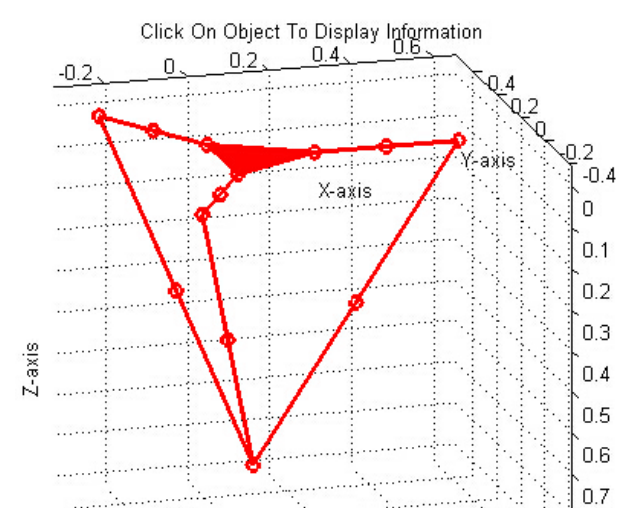

Fig.7 Three dimensional model (MATLAB) 


$$
\begin{aligned}
& u_{i}=\left(\begin{array}{lll}
\cos \beta_{i} \cos \theta_{i} & \sin \beta_{i} \cos \theta_{i}-\sin \theta_{i}
\end{array}\right)^{T} \\
& \theta_{i}=2 \arctan \frac{-E_{i}-\sqrt{E_{i}^{2}-G_{i}^{2}+F_{i}^{2}}}{G_{i}-F_{i}}, i=1,2,3 \\
& E_{i}=2 l_{1}\left(r-e_{i}\right)^{T}\left[\begin{array}{lll}
0 & 0 & 1
\end{array}\right] \\
& F_{i}=2 \sigma_{X}^{2}\left(r-e_{i}\right)^{T}\left(\cos \beta_{i} X+\sin \beta_{i} Y\right), X=\left[\begin{array}{lll}
1 & 0 & 0
\end{array}\right], Y=\left[\begin{array}{lll}
1 & 0 & 0
\end{array}\right] \\
& G_{i}=\left(r-e_{i}\right)^{T}\left(r-e_{i}\right)+l_{1}^{2}-l_{2}{ }^{2} \\
& r-e_{i}-l_{1} u_{i}=l_{2} w_{i}, i=1,2,3 \\
& \left(r-e_{i}-l_{1} u_{i}\right)^{T}\left(r-e_{i}-l_{1} u_{i}\right)=l_{2}{ }^{2} \\
& E_{i} \sin \theta_{i}+F_{i} \cos \theta_{i}+G_{i}=0, i=1,2,3
\end{aligned}
$$

The formula (1) expressed the position vector. The mold of $e_{i}$ said the circumscribed circle radius difference between the static platform and moving platform. Namely, O to vector in the Fig.6.It expressed the static Angle of platform structure which were the active arm of branched chain respectively and the slave arm length and unit vector. The formula (5) expressed the active arm Angle. This Angle can be used as the basis of a drive motor rotation. The formula(1) turned to (9).It multiplied by the respective on both ends of transposed, and it got (10), the formula (4) into the formula (10), and it got the formula(11) .By expressed the formula (6), (7), (8), the reduction of the formula(11)which can be the initiative arm Angle expression of the formula (5).

\section{The modeling of three degree of freedom parallel manipulator}

In MATLAB of SIMULINK module, the modeling of three degree of freedom parallel manipulator is set up ${ }^{[6]}$.It is as shown in Fig. 7.

1) With the aid of MATLAB SimMechanics in the toolbox, the process of Modeling is finished. The toolkit provides a number of corresponding to the actual system components. Using these modules can be convenient to create diagrams to model complex mechanical system. These modules can analysis mechanical system alone or in combination with any of the controllers designed by SIMULINK, and other comprehensive simulation of dynamic system are linked together.

2) As shown in the triangle of the Fig.7, parallel manipulator moving platform is simplified as a bit of static platform, and active arm driven arm can be clearly represented. By SimMechanics toolkit active to static platform, we can also set the arm, the quality of the slave arm, size, initial position in order to a better simulation.

\section{Trajectory planning}

The three DOF parallel manipulator tasks is capture, transfer, placement, taking into account the operation of the process of a certain height or width of the obstacle, So we put the motion path to the door shape. As shown in Fig. 8. Therefore, a reasonable arc excessive model becomes the ideal choice of the manipulator motion path. According to the principle of the shorter path first, we set the motion trajectory of manipulator for Modified trapezoidal:

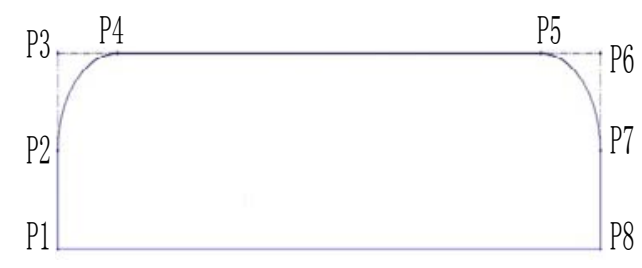

Fig. 8 Trajectory planning

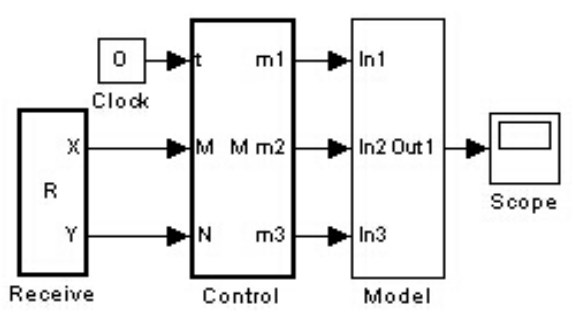

Fig. 9The simulation module 
1) The trajectory of three degrees of freedom parallel manipulator projection in the Y-axis direction is $\overrightarrow{p 3 p 6}$; X-axis direction are $\overrightarrow{p 1 p 3}$ and $\overrightarrow{p 6 p 8}$. The starting point coordinates of movement track of the three DOF parallel manipulator is random, but the end point of coordinate trajectory is fixed. Because the mechanical hand placed the object position is fixed, so the end point coordinates of coordinate manipulator motion trajectory is fixed. The basic trajectory of the three-DOF parallel manipulator is $\overrightarrow{p 1 p 2} \rightarrow \overrightarrow{p 2 p 4} \rightarrow \overrightarrow{p 4 p 5} \rightarrow \overrightarrow{p 5 p 7} \rightarrow \overrightarrow{p 7 p 8}$, as in Fig.8.

2) After the motion controller to get the coordinates of the target object from VBAI software, quickly moved to the coordinate position. We set up three-DOF parallel manipulator with a pick and place tasks to complete are 3 seconds. $\overrightarrow{p 1 p 2}$ segment movement time is $0.5 \mathrm{~s} ; \quad \overrightarrow{p 2 p 4}$ segment movement time is $\quad 0.5 \mathrm{~s} ; \quad \overrightarrow{p 4 p 5} \quad$ segment movement time $\quad$ is $1 \mathrm{~s} ; \quad \overrightarrow{p 5 p 7}$ segment movement time is $0.5 \mathrm{~s} ; \overrightarrow{p 7 p 8}$ segment movement time is $0.5 \mathrm{~s}$. Assuming the robot crawl objects need to upgrade the height is $50 \mathrm{~mm}$. The coordinate of the target object is $(\mathrm{M}, \mathrm{N})$, the coordinates of the placement point is $(100,200,-850)$. We can plan a robot moving platform at each interval movement trajectory is:

(1) $\overrightarrow{p 1 p 2}: 0<\mathrm{t} \leq 0.5 \mathrm{~s}$

The direction of $\mathrm{X}$ axis: $\mathrm{M}$; The direction of $\mathrm{Y}$ axis: $\mathrm{N}$;

The direction of $\mathrm{Z}$ axis: $-850+50(\mathrm{t}-(1 \div 2 \pi) \times \sin (2 \pi \mathrm{t}))$.

(2) $\overrightarrow{p 2 p 4}: 0.5 \mathrm{~s}<\mathrm{t} \leq 1 \mathrm{~s}$

The direction of $\mathrm{X}$ axis: $M+\frac{100-\mathrm{M}}{200-\mathrm{N}} \times 50\left((\mathrm{t}-0.5)-\frac{1}{4 \pi} \times \sin (4 \pi(t-0.5))\right)$;

The direction of $\mathrm{Y}$ axis: $\mathrm{N}+50\left((\mathrm{t}-0.5)-\frac{1}{4 \pi} \sin (4 \pi(\mathrm{t}-0.5))\right)$;

The direction of $\mathrm{Z}$ axis: $-850+50\left(\mathrm{t}-\frac{1}{2 \pi} \sin (2 \pi \mathrm{t})\right)$.

(3) $\overrightarrow{p 4 p 5}: 1 \mathrm{~s}<\mathrm{t} \leq 2 \mathrm{~s}$

The direction of $\mathrm{X}$ axis: $\mathrm{M}+\frac{100-\mathrm{M}}{200-\mathrm{N}} \times 25+\frac{100-\mathrm{M}}{200-\mathrm{N}} \times(200-\mathrm{N}-50) \times\left((\mathrm{t}-1)-\frac{1}{2 \pi} \times \sin (2 \pi(\mathrm{t}-1))\right)$;

The direction of $\mathrm{Y}$ axis: $\mathrm{N}+25+(200-\mathrm{N}-50)\left((\mathrm{t}-1)-\frac{1}{2 \pi} \sin (2 \pi(\mathrm{t}-1))\right)$;

The direction of $\mathrm{Z}$ axis:-800.

(4) $\overrightarrow{p 5 p 7}: 2 \mathrm{~s}<\mathrm{t} \leq 2.5 \mathrm{~s}$

The direction of $X$ axis: $M+\frac{100-M}{200-N}\left(200-N-\frac{100-M}{200-N} \times 50\right)+\frac{100-M}{200-N} \times 50\left((t-2)-\frac{1}{4 \pi} \sin (4 \pi(t-2))\right)$;

The direction of $\mathrm{Y}$ axis: $(\mathrm{N}+(200-\mathrm{N}-25))+50\left((\mathrm{t}-2)-\frac{1}{4 \pi} \sin (4 \pi(\mathrm{t}-2))\right)$;

The direction of $\mathrm{Z}$ axis: $-800-50\left((\mathrm{t}-2)-\frac{1}{2 \pi} \sin (2 \pi(\mathrm{t}-2))\right)$.

(5) $\overrightarrow{p 7 p 8}: 2.5 \mathrm{~s}<\mathrm{t} \leq 3 \mathrm{~s}$

The direction of $\mathrm{X}$ axis:100 ; The direction of Y axis:200; 
The direction of $\mathrm{Z}$ axis: $-800-50\left((\mathrm{t}-2)-\frac{1}{2 \pi} \sin (2 \pi(\mathrm{t}-2))\right)$.

\section{5. the MATLAB simulation}

In the MATLAB of SIMULINK module, the establishment of the model is as shown in Fig. 9. The model is set of three parts: the serial data receiving part, the robot motion control parts and mechanical manipulator model. VBAI and MATLAB can be established through a serial port communication. It will obtain the coordinates of the object under fetching VBAI passed to the manipulator and give the coordinates of the manipulator $(\mathrm{M}, \mathrm{N})$ in the assignment. The height of the $\mathrm{Z}$ direction changing [- $850 \mathrm{~mm}, 850 \mathrm{~mm}$ ].The design of the simulation experiments of three groups of different grab position which is taking objects obtained respectively by VBAI coordinates $(-100$, -200), (-200, -150), (-180, -230). The moving platform in the process of fetching manipulator trajectory as shown in Fig. 10 to 12.It can be seen from the Fig.. According to certain manipulator trajectory by $(\mathrm{M}, \mathrm{N})$ coordinates movement comes to an end position $(100,200)$.

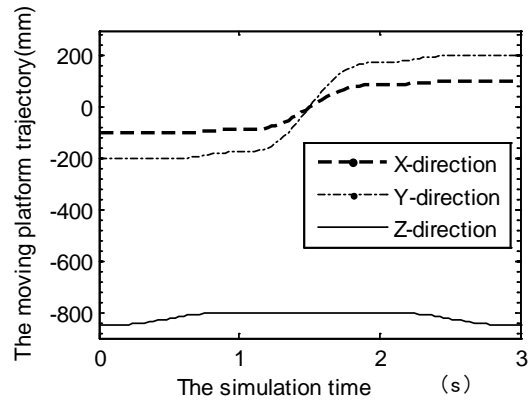

Fig. $10(\mathrm{M}, \mathrm{N})$ is $(-100,-200)$

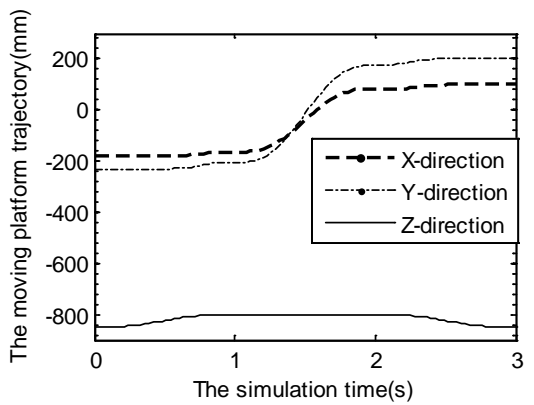

Fig. 12(M, N)is (-180, -230)

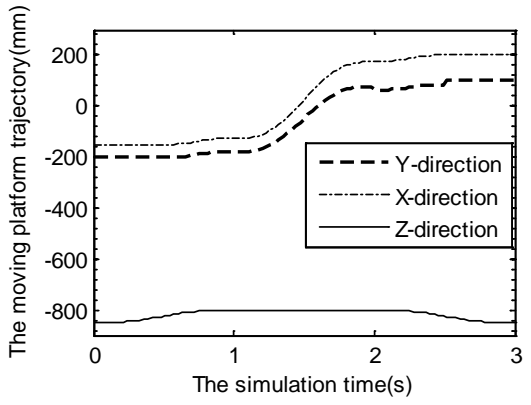

Fig. 11（M, N) is $(-200,-150)$

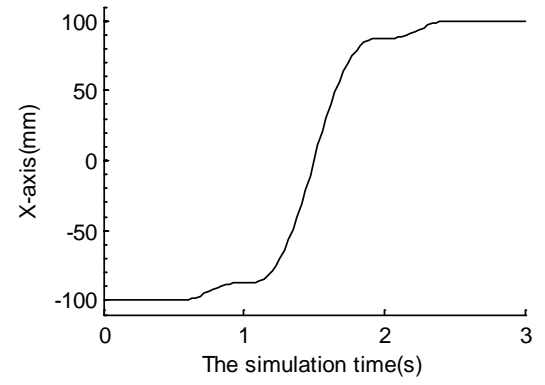

Fig. 13Trajectory (X-direction)

As the coordinates for fetching things (-100, -200,-850), the value of $\mathrm{M}$ and $\mathrm{N}$ are respectively- 100 , - 200.Mechanical hand platform is respectively in the $\mathrm{X}, \mathrm{Y}, \mathrm{Z}$ direction of the trajectory as shown in Fig. 13 and 15. The moving of the plat form Motion trajectory should be a straight line. Because it is a straight line in the XY plane target point and end point of the shortest path .As shown in Fig. 16, it is a straight line which is driven platform motion trajectory in space in the XY plane projection. As shown in Fig. 17, the three-dimensional space observed the actual trajectory of moving platform modified trapezoidal.

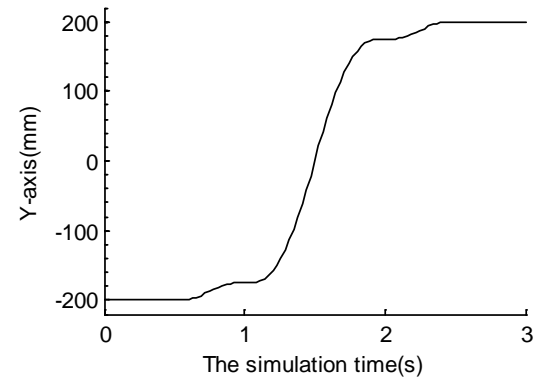

Fig. 14 Trajectory (Y-direction)

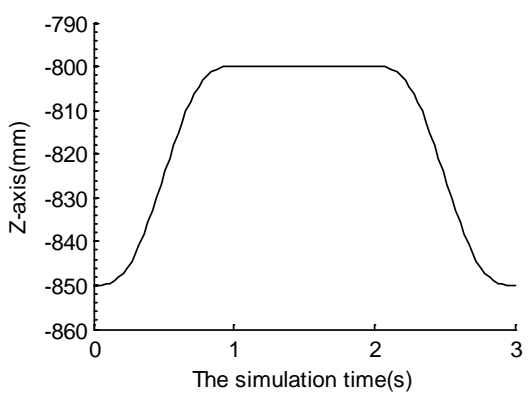

Fig. 15 Trajectory (Z-direction) 


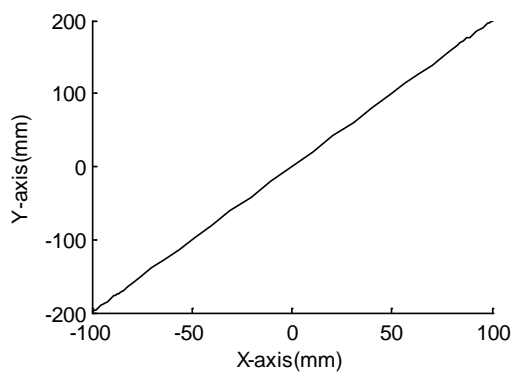

Fig. 16 Trajectory(XY-plane)

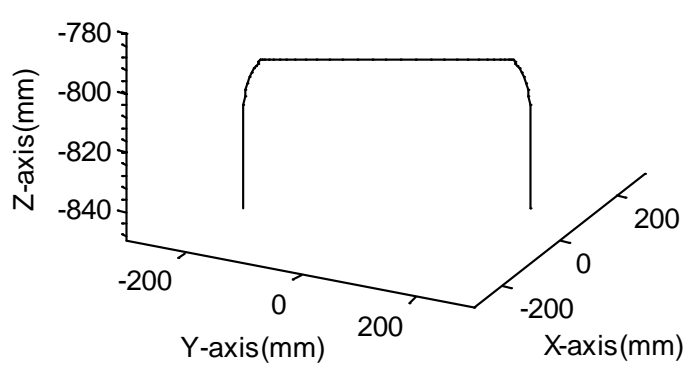

Fig. 17 Trajectory(Three-dimensional space)

\section{Summary}

For three degree of freedom parallel manipulator system, this paper uses the VBAI software technology target. The MATLAB software is used for trajectory planning modeling and mechanical structure. It has carried on the VBAI and the combination of MATLAB software simulation proved that the parallel manipulator system meets the design requirements of accuracy and rapidity. The paper proves the joint simulation method is effective.

\section{Acknowledgements}

This paper is supported by Institute of electrical engineering, Hebei University of Science and Technology and Institute of Applied Mathematics, Hebei Academy of Sciences.

\section{Corresponding author}

Chaoying Liu, E-mail: liucy@hebust.edu.cn.

\section{References}

[1] GOSSELIN C. Determination of the workspace of 6-DOF parallel manipulators [J]. Journal of Mechanical Design, 1990, 12(3):331-335.

[2] KEMALS, KORKMAZO, Trajectory tracking control of parallel robots in the presence of joint drive flexibility [J]. Journal of Sound and Vibration, 2009, 319:77-90

[3] Clavel R. Delta, a fast robot with parallel geometry[C]. Proceedings of 18th International Symposium on Industrial Robots, Sydney, Australia, 1988: 91-100

[4] Clavel R, Dispositif pour le deplacement et le positionnement d'un element dans l'espace, Switzerland patent, CH1985005348856, 1985

[5] Clavel R, Device for the movement and positioning of an element in space, U.S. Patent, No. 4,976,582, 1990

[6] Z.Zhen,C.Y.Liu,Y.K.Zhang andC.M.Hao, The Combined Simulation of High-speed Parallel Manipulator based on MATLAB, SolidWorks and ADAMS, Mechanical Engineering and Materials Science.Taiyuan,2014,1578-1581 